\title{
ON SOME SPACES OF SUMMABLE SEQUENCES AND THEIR DUALS
}

GERALDO SOARES DE SOUZA and G. O. GOLIGHTLY

Department of Mathematics

Auburn Univers1ty

Alabama 36849

(Received May 18, 1984 and Revised March 11, 1985)

ABSTRACT. Suppose that $S$ is the space of all summable sequences $\alpha$ with $\|\alpha\|_{S}=\sup _{n \geq 0}\left|\sum_{j=n}^{\infty} \alpha_{j}\right|$ and $J$ the space of all sequences $B$ of bounded varlation with $\| \beta_{J}=\left|\beta_{0}\right|+\sum_{j=1}^{\infty}\left|\beta_{j}-\beta_{j-1}\right|$. Then for $\alpha$ in $S$ and $\beta$ in $J$ $\left|\sum_{j=0}^{\infty} \alpha_{j} \beta_{j}\right| \leq\|\alpha\|_{S}\|\beta\|_{J}$; this inequality leads to the description of the dual space of $S$ as $J$. It, related Inequalitles, and their consequences are the content of this paper. In particular, the inequality cited above leads directly to the Stolz form of Abel's theorem and provides a very simple argument. Also, some other sequence spaces are discussed.

KEY WORDS AND PHRASES. DUal spaces, sequence spaces, bounded operators. 1980 Mathematics Subject Classification: 46A45, 46A20

1. INTRODUCTION.

Suppose $S$ is the space of all summable sequences $s=\left(s_{n}\right)_{n=0}^{\infty}$, with norm given by $\|s\|_{S}=\operatorname{Sup}_{n>0}\left|\sum_{j=n}^{\infty} s_{j}\right|$, the remainder in the sum of $s$ after n-terms. An example of such an $\bar{s}$, summable but not absolutely summable, is given by $s_{n}=\frac{(-1)^{n}}{n+1}, n=0,1,2,3, \ldots$ so that $\sum_{j=0}^{\infty} \frac{(-1)^{n}}{n+1}$ is an alternating convergent series. Here, as is readily verified, $\|s\|_{s}=\operatorname{Sup}_{n>0}\left|\sum_{j=n}^{\infty} \frac{(-1)^{j}}{j+1}\right|=\ln 2$, the the natural $\log$ of 2 . This paper is based on the observation that $S$, with this norm, is naturally isomorphic to $c_{0}$, the space of all sequences having $11 \mathrm{mit}$ zero endowed with the supremum norm. Moreover, if we consider $S$ on 1 ts own, we get several interesting results. For example, since $S$ is isomorphic to $c_{0}$ and $c_{0}$, the dual of $c_{0}$, is 1somorphic to $\ell_{1}$, the space of all absolutely summable sequences, then 1 follows that $S^{*}$, the dual of $S$, is isomorphic to $\ell_{1}$. 
However, if we compute the dual of $S$ without using the isomorphism with $c_{0}$, we find that $S *$ is isomorphic to $J$, the space of all sequences $B$ of bounded variation with

$\|\beta\|_{J}=\left|\beta_{0}\right|+\sum_{n=1}^{\infty}\left|\beta_{n}-\beta_{n-1}\right| ;$ an example of such a $\beta$ being given by $\beta_{n}=\frac{1}{n+1} n=0,1,2,3, \ldots$ so that $\|\beta\|_{J}=1+\sum_{n=1}^{\infty}\left[\frac{1}{n}-\frac{1}{n+1}\right]=2$. of course, $J$ is isomorphic to $\ell_{1}$. Another example is that we get the following new inequality: If $B \in J$ and $s \in S\left|\sum_{j=0}^{\infty} s_{j} \beta_{j}\right|<\|s\|_{S} \cdot\|\beta\|_{J}$, which can be used to give a proof of Abel's Continuity Theorem.

Similarly, we consider the space $H$ of all sequences $\lambda=\left(\lambda_{n}\right)_{n=0}^{\infty}$ such that $\|\lambda\|_{H}=\sup _{n>0}\left|\sum_{j=0}^{n} \lambda_{j}\right|$ is finite; for example $\lambda_{n}=(-1)^{n}, n=0,1,2,3, \ldots$, so that $\|\lambda\|_{H}=1$. We get the following inequality: If $\lambda \varepsilon H, \beta \varepsilon \mathrm{J}$, and $\rho=1 \mathrm{~m} B$ then $\left|\lambda_{0} \rho+\sum_{n=1}^{\infty} \lambda_{n}\left(\rho-\beta_{n-1}\right)\right| \leq\|\lambda\|_{H}\|\| \beta \|_{J}$. Consequently we have that $\phi \varepsilon J *$ if and only if $\phi(\beta)=\lambda_{0} \rho+\sum_{n=1}^{\infty} \lambda_{n}\left(\rho-\beta_{n-1}\right)$ for $\beta \varepsilon J$.

Also, we give characterizations of the bounded linear operators on $S$ easily obtained from a description of operators on $c_{0}$. These results, however, may be obtained using the uniform boundedness principle.

To make the presentation reasonably self-contained, we shall include a resume of pertinent results and definitions.

\section{PRELIMINARIES}

DEFINITION 2.1. Let $\left(X,\|\|_{X}\right)$ and $\left(Y,\|\|_{Y}\right)$ be two sequence spaces, which are Banach spaces with the respective norms. For a fixed $y=\left(y_{n}\right) \varepsilon Y$ define the mapping $\phi_{y}: x \rightarrow R$ by $\phi_{y}(x)=\sum_{n=0}^{\infty} x_{n} y_{n}$ for those $x=\left(x_{n}\right)$ in $x$ for which the series converges.

Assume that $\left|\phi_{y}(x)\right| \leq M\left\|_{x}\right\|_{X}$ with $M$ some absolute constant, that is, $\phi_{y}$ is a bounded linear functional on $X$ for any fixed $y \in Y$. Let $X *$ be the space of all bounded linear functionals $\phi$ on $X$, endowed with norm $\|\phi\|_{X^{*}}=\sup _{\|\mathrm{X}\|_{X}=1}|\phi(x)|$ and $\psi$ the mapping from $Y$ to $X *$ given by $\psi(y)=\phi_{y}$. If $\psi$ is onto and $\|y\|_{Y}=\left\|\phi_{Y}\right\|_{X *}$, then we say that $Y$ is the dual of $X$, in this sense we write $Y=X^{*}$.

DEFINITION 2.2. We define the sequence spaces $S, \ell_{1}, \ell_{\infty}, c_{0}$, and $H$ respectively by

$$
\begin{aligned}
& s=\left\{s=\left(s_{n}\right)_{n=0}^{\infty}, \text { summable sequences such that }\|s\|_{s}=\sup _{p \geq 0}\left|\sum_{j=p}^{\infty} s_{j}\right|\right\}, \\
& \ell_{1}=\left\{\delta=\left(\delta_{n}\right)_{n=0}^{\infty},\|\delta\|_{\ell_{1}}=\sum_{j=0}^{\infty}\left|\delta_{j}\right|<\infty\right\}, \\
& \ell_{\infty}=\left\{\mu=\left(\mu_{n}\right)_{n=0}^{\infty},\|\mu\|_{\ell_{\infty}}=\sup _{j \geq 0}\left|\mu_{j}\right|<\infty\right\}, \\
& c_{0}=\left\{\gamma=\left(\gamma_{n}\right)_{n=0}^{\infty}, \prod_{n \rightarrow \infty} \gamma_{n}=0,\|\gamma\|_{c_{0}}=\underset{\substack{n \geq 0 \\
n}}{\sup }\left|\gamma_{n}\right|\right\}
\end{aligned}
$$




$$
\begin{aligned}
& H=\left\{\lambda=\left(\lambda_{n}\right)_{n=0}^{\infty},\|\lambda\|_{H}=\sup _{n \geq 0}\left|\sum_{j=0}^{n} \lambda_{j}\right|<\infty\right\} . \\
& \text { We believe that this is the first time that } S \text { is taken as a space with this }
\end{aligned}
$$
norท.

TIF IREY 2.3. The space $S$ is a Banach space, isometrically isomorphic to $c_{0}$. The isometry $\mathrm{T}: \mathrm{S}+\mathrm{c}_{0}$ being given by $\mathrm{T}(\mathrm{s})=\left(\mathrm{r}_{\mathrm{n}}(\mathrm{s})\right)_{\mathrm{n}=0}^{\infty}$ and $\mathrm{T}^{-1}: \mathrm{c}_{0}+\mathrm{s}$ by $r^{-1}(r)=\left(\gamma_{n}-\gamma_{n+1}\right)_{n=0}^{\infty}$, where $r_{n}(s)=\sum_{j=n}^{\infty} \alpha_{j}$.

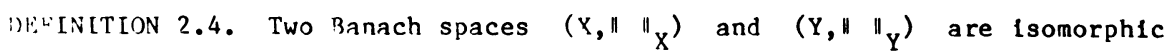
eqilvalents if there exist a one-to-one and onto linear mapping $T: X+Y$ such that $v\|:\| \mathrm{Y} \leq\|\mathrm{TX}\| \mathrm{Y} \leq \mathrm{M}\|\mathrm{X}\| \mathrm{X}$ with $\mathrm{N}$ and $\mathrm{Y}$ absolute constants. If there exists such a such a $T$ with $\|T x\|_{Y}=\|x\|_{X}$, then we say that $X$ and $Y$ are isometrically isonorphic.

Notice in Theorem $2.3 \mathrm{~T}$ and $\mathrm{T}^{-1}$ can be represented as infinite matrices,

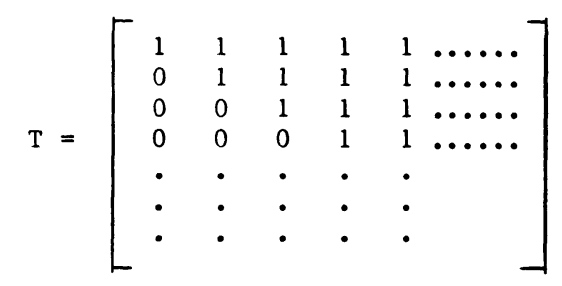

$$
\begin{aligned}
& \mathrm{T}^{-1}=\left[\begin{array}{rrrrrrr}
1 & -1 & 0 & 0 & 0 & \ldots & \ldots \\
0 & 1 & -1 & 0 & 0 & \ldots & \ldots \\
0 & 0 & 1 & -1 & 0 & \ldots & \ldots \\
0 & 0 & 0 & 1 & -1 & 0 & \ldots \\
. & . & . & . & . & & \\
\cdot & . & . & . & . & & \\
\cdot & . & . & . & . & &
\end{array}\right]
\end{aligned}
$$

THEOREM 2.5. $M$ is a bounded linear operator from $c_{0}$ into $c_{0}$ if and only if $M$ is represented by an infinite matrix with columns in $c_{0}$ and rows in $\ell_{1}$, uniformly bounded in $\ell_{1}$. That is if $M=\left(m_{n k}\right)$ then
1) $\lim _{n \rightarrow \infty} m_{n k}=0$ for any fixed $k$,
11) $\sup _{n \geq 0} \sum_{k=0}^{\infty}\left|m_{n k}\right|<\infty$.

THEOREM 2.6. $\phi$ is in $c_{0}{ }^{*}$ if and only if there is a $\delta=\left(\delta_{n}\right) \varepsilon l_{1}$ such that for any $\gamma=\left(\gamma_{n}\right) \varepsilon c_{0} \phi(\gamma)=\sum_{n=0}^{\infty} \delta_{n} \gamma_{n}$, in which case there is only one such $\delta=\left(\delta_{n}\right) \varepsilon \ell_{1}$ and $\|\phi\|_{c_{0} *}=\|\delta\|_{\ell_{1}}$. 
THEORE:1 2.7. The space $J$ is a Banach space, isometrically 1somorphic to $\ell_{1}$. The isometry $L: J+\ell_{1}$ is given by $L(\beta)=\left(\beta_{n}-\beta_{n-1}\right)_{n=0}^{\infty}$, $B_{-1}=0$, and $L^{-1}: l_{1}+J$ by $L^{-1}(\delta)=\left(\sum_{j=0}^{n} \delta_{j}\right)_{n=0}^{\infty}$ with $\delta=\left(\delta_{n}\right) \varepsilon l_{1}$ and $\beta=\left(\beta_{n}\right) \varepsilon J$.

THEOREM 2.8. The space $H$ is a Banach space Isometrically isomorphic to $l_{\infty} \cdot$ The isometry $\psi: H \rightarrow \ell_{\infty}$ is given by $\psi(\lambda)=\left(\sum_{j=0}^{n} \lambda_{j}\right)_{n=0}^{\infty}$ and $\psi^{-1}: \ell_{\infty}+H$ by $\psi^{-1}(\mu)=\left(\mu_{n}-\mu_{n-1}\right)_{n=0}^{\infty}$ with $\mu_{-1}=0, \mu=\left(\mu_{n}\right) \varepsilon \ell_{\infty}, \lambda=\left(\lambda_{n}\right) \varepsilon H$.

3. THE DUAL OF $S$

THEOREM 3.1 (Holder's type inequality). If $s=\left(s_{n}\right) \varepsilon s$ and $\beta=\left(\beta_{n}\right) \varepsilon J$ then $\left|\sum_{n=0}^{\infty} s_{n}{ }_{n}\right| \leq\|\beta\|_{J} \cdot\|s\|_{s}$.

Proof: For $N \geq 1$, we notice that

$$
\left|\sum_{n=0}^{N-1} s_{n} B_{n}-r_{0}(s) B_{0}-\sum_{n=1}^{N} r_{n}(s)\left[B_{n}-B_{n-1}\right]\right|=\left|r_{N}(s) B_{N}\right|=\left|r_{N}(s)\right| \cdot\left|B_{N}\right| \text {, }
$$

where $r_{n}(s)=\sum_{j=n}^{\infty} s_{j}$. Now since $s$ is summable and $B$ is bounded, it follows that $\left|r_{v}(s)\right|\left|\beta_{N}\right| \rightarrow 0$ as $N \rightarrow \infty$. Therefore we have proved that

$$
\begin{gathered}
\sum_{n=0}^{\infty} s_{n} \beta_{n}=r_{0}(s) \beta_{0}+\sum_{n=1}^{\infty} r_{n}(s)\left[\beta_{n}-\beta_{n-1}\right] \text {; consequently } \\
\left|\sum_{n=0}^{\infty} s_{n} \beta_{n}\right| \leq \sup _{n \geq 0}\left|r_{n}(s)\right|\left(\left|\beta_{0}\right|+\sum_{n=1}^{\infty}\left|\beta_{n}-\beta_{n-1}\right|\right),
\end{gathered}
$$

so that the theorem is proved.

Consider the mapping $\phi_{B}: S \rightarrow R$ defined by $\phi_{B}(s)=\sum_{n=0}^{\infty} s_{n} \beta_{n}$ where $\beta=\left(\beta_{n}\right)$ is a fixed element in $J$. In view of Theorem 3.1 , we have $\phi_{B}$ is a bounded linear functional on $S$. At this point, a natural question is: are these all the bounded linear functionals on $S$ ? The next result tells us that the answer is yes.

THEOREM 3.2. (Duality Theorem). If $\phi \in S$ then there is a unique $\beta=\left(\beta_{n}\right) \in J$ such that $\phi=\phi_{\beta}$, that is, $\phi(s)=\sum_{n=0}^{\infty} s_{n} \beta_{n}$ for any $s=\left(s_{n}\right) \varepsilon s$. Moreover $\|\phi\|_{S^{*}}=\|\beta\|_{\mathrm{J}^{*}}$. Conversely if $\phi(s)=\sum_{n=0}^{\infty} s_{n^{\beta}}{ }_{n}$ then $\phi \varepsilon S^{*}$ so that the mapping $\psi: J \rightarrow S^{*}$ defined by $\psi(B)=\phi_{B}$ is an isometric isomorphism from $J$ to $S^{*}$. Proof: If $\phi(s)=\sum_{n=0}^{\infty} s_{n}{ }^{\beta}$, then we already have seen by Theorem 3.1 that $\phi$ is a bounded linear functional on $S$, that is $\phi \varepsilon S^{*}$. So it remains to prove the first part. Let $\phi \varepsilon S^{*}$; then using Theorem 2.3, we have

$$
\phi(s)=\phi\left(T^{-1} \circ T(s)\right)=\left(\phi \circ T^{-1}\right)(T(s))=\left(\phi \circ T^{-1}\right)\left(\left(\sum_{j=n_{j}^{s}}^{\infty}\right)_{n=0}^{\infty}\right) \text {. }
$$


Now autice that $\phi \circ \mathrm{T}^{-1} \varepsilon \mathrm{c}_{0}$, so that there is a $\mathrm{y}=\left(\mathrm{y}_{\mathrm{n}}\right) \varepsilon \ell_{1}$ (see Theorem 2.6) such that

$$
\phi(s)=\left(\phi \circ T^{-1}\right)\left(\left(\sum_{j=n}^{\infty} s_{j}\right)_{n=0}^{\infty}\right)=\sum_{n=0}^{\infty} y_{n}\left(\sum_{j=n}^{\infty} s_{j}\right) \text { and }\left\|\circ \circ T^{-1}\right\|_{c_{0}}=\|y\| l_{\ell_{1}} .
$$

Observe that (3.3) can be written $\phi(s)=\sum_{n=0}^{\infty} s s_{n_{j}} \sum_{j}^{n} y_{j}$; therefore we may let $B=\left(B_{n}\right)$ where $B_{n}=\sum_{j=0}^{n} y_{j}$.

Since $\beta=\mathrm{L}^{-1}(\mathrm{y}), \quad B$ is in $\mathrm{J}$. Consequently given $\phi \varepsilon \mathrm{S}^{*}$ there is a $\beta \varepsilon J$ such that $\phi=\phi_{B}$. On the other hand Theorem 2.6 tells us that

$\left\|\phi \circ \mathrm{T}^{-1}\right\| \mathrm{c}_{0} *=\sum_{\mathrm{n}=0}^{\infty}\left|\mathrm{y}_{\mathrm{n}}\right|$; therefore $\|\beta\|_{\mathrm{J}}=\sum_{\mathrm{n}=0}^{\infty}\left|\mathrm{y}_{\mathrm{n}}\right|=\left\|\phi \circ \mathrm{T}^{-1}\right\| \mathrm{c}_{0} * \leq\left\|\mathrm{T}^{-1}\right\| \cdot\|\phi\|_{S *}$

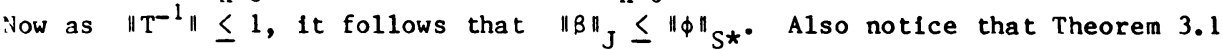
implies $\|\phi\|_{S *} \leq\|\beta\|_{J}\left(\right.$ since $\left.\phi=\phi_{\beta}\right)$. Therefore putting together these last two inequalities we have $\|\phi\|_{S^{*}}=\|\beta\|_{J}$; consequently the mapping $\psi: J \rightarrow S^{*}$ defined by $\psi(\beta)=\phi_{3}$ is an 1sometry, so that the theorem is proved.

We have used Theorem 3.1 to characterize all bounded linear functionals on S. Now we are going to use this theorem again to give a proof of Abel's continulty theorem which is as follows.

THEOREM 3.4 (Abel's continuity theorem). If $\alpha=\left(\alpha_{n}\right)$ is summable and $f(z)=\sum_{n=0}^{\infty} \alpha_{n} z^{n}$, then $\lim _{z \rightarrow 1} f(z)=f(1)$, where $z$ is restricted to approach the point 1 in such a way that $|z|<1$ and $\frac{|1-z|}{1-|z|}$ remains bounded.

Proof: First of all, let $C$ be a positive absolute constant such that $\frac{|1-z|}{1-|z|} \leq \mathrm{c}$ and $|\mathrm{z}|<1$. Notice that $\left|\sum_{\mathrm{p}=\mathrm{N}}^{\infty} \alpha_{\mathrm{p}} \mathrm{z}^{\mathrm{p}}\right| \leq n\left(\mathrm{z}^{\mathrm{p}}\right)_{\mathrm{p}=\mathrm{N}}^{\infty} \mathrm{NJ} \cdot \|\left(\alpha_{\mathrm{p}}\right)_{\mathrm{p}=\mathrm{N}}^{\infty} \mathrm{s}$ for $N \geq 1$ by the above Inequality applied to the sequences $\left(\alpha_{p}\right)$ and $\left(z^{p}\right)$, since $\left(z^{p}\right)_{p=N}^{\infty}$ is in $J$; in fact, since $|z|<1$

$$
\begin{aligned}
& \left\|\left(z^{p}\right)_{p=N}^{\infty}\right\|_{J}=|z|^{N}+\left|z^{N}-z^{N+1}\right|+\left|z^{N+1}-z^{N+2}\right|+\ldots . .= \\
& =|z|^{N}+|1-z|\left(|z|^{N}+|z|^{N+1}+\ldots . .\right)=|z|^{N}\left(1+\mid \frac{1-z}{1-|z|}\right) \text {. }
\end{aligned}
$$

Now using the hypothesis we have $\|\left(z^{\mathrm{p}}\right)_{\mathrm{p}=\mathrm{N}^{\sharp} \mathrm{J}}^{\infty} \leq 1+\mathrm{C}$. Consequently, $\left|\sum_{\mathrm{p}=\mathrm{N}}^{\infty} \alpha_{\mathrm{p}} \mathrm{z}^{\mathrm{p}}\right| \leq(1+\mathrm{C}) \|\left(\alpha_{\mathrm{p}}\right)_{\mathrm{p}=\mathrm{N}}^{\infty} \mathrm{s} \rightarrow 0$ as $\mathrm{N} \rightarrow \infty$ since $\left(\alpha_{\mathrm{n}}\right) \varepsilon \mathrm{s}$. Then $\left|\sum_{p=0}^{\infty} \alpha_{p} z^{p}-\sum_{p=0}^{\infty} \alpha_{p}\right| \leq\left|\sum_{p=0}^{N-1} \alpha_{p} z^{p}-\sum_{p=0}^{N-1} \alpha_{p}\right|+\left|\sum_{p=N}^{\infty} \alpha_{p} z^{p}-\sum_{p=N}^{\infty} \alpha_{p}\right|=A+B$. For $\varepsilon>0$, fix $N$ so that $B \leq(2+C)\left\|\left(\alpha_{p}\right)_{p=N}^{\infty}\right\| S \leq \varepsilon / 2$. For $|z-1|$ sufficiently small, $A<\varepsilon / 2$. Hence $\lim _{z \rightarrow 1} \sum_{p=0}^{\infty} \alpha_{p} z^{p}=\sum_{p=0}^{\infty} \alpha_{p}$. Therefore the theorem is proved. 
4. THE DUAL OF J

of course, for fixel $s=\left(s_{n}\right)$ in $s$ the formula $\phi_{s}(\beta)=\sum_{j=0}^{\infty} s_{j} \beta_{j}$ defines a bounded linear functional on $J$. However, these are not all the members of $J^{*}$. As a key to our description of $J^{*}$, we note that if $\beta=\left(\beta_{n}\right)$ is in $J$ with 1 imit $\rho$ then $\phi_{s}(\beta)=\left(\sum_{j=0}^{\infty} s_{j}\right) \rho+\sum_{j=0}^{\infty} s_{j}\left(\beta_{j}-\rho\right)=$ $\lambda_{0} \rho+\sum_{j=1}^{\infty} \lambda_{j}\left(\rho-\beta_{j-1}\right)$, where $\lambda_{0}=\sum_{j=0}^{\infty} s_{j}$ and for $j \geq 1 \lambda_{j}=-s_{j-1}$. llere $\sum_{j=0}^{\infty} \lambda_{j}=0$; so that $\lambda$ is in $S$.

THEOREM 4.1 (Holder's type inequality). If $\lambda=\left(\lambda_{n}\right) \varepsilon H, \beta=\left(\beta_{n}\right) \varepsilon J$, and $\rho=11 m B$ then $\left|\lambda_{0} \rho+\sum_{n=1}^{\infty} \lambda_{n}\left(\rho-\beta_{n-1}\right)\right| \leq\|\lambda\|_{H} \cdot\|B\|_{J}$.

Proof: This Inequality follows easily from the observation that $\lambda_{0} \rho+\sum_{n=1}^{\infty} \lambda_{n}\left(\rho-\beta_{n-1}\right)=\lambda_{0} \beta_{0}+\sum_{n=1}^{\infty}\left(\sum_{j=0}^{n} \lambda_{j}\right)\left[\beta_{n}-\beta_{n-1}\right]$; so that $\left|\lambda_{0} \rho+\sum_{n=1}^{\infty} \lambda_{n}\left(\rho-\beta_{n-1}\right)\right| \leq \sup _{n>0}\left|\sum_{j=0}^{n} \lambda_{j}\right|\left(\left|\beta_{0}\right|+\sum_{n=1}^{\infty}\left|\beta_{n}-\beta_{n-1}\right|\right)$. As we did for $S$, we consider the mapping $\phi_{\lambda}: J+R$ defined by $\phi_{\lambda}(\beta)=\lambda_{0} \rho+\sum_{n=1}^{\infty} \lambda_{n}\left(\rho-\beta_{n-1}\right)$ where $\lambda=\left(\lambda_{n}\right)$ is a fixed element in $H$. Theorem 4.1 assures us that $\phi_{\lambda}$ is a bounded linear functional on $J$. The claim of the next theorem is that these are all the bounded linear functionals on $\mathrm{J}$. Here, our representation is in a slightly different sense than that of the Definition 2.1, the latter appearing more natural to us.

THEOREM (4.2) (Duality Theorem). If $\phi \varepsilon J *$ then there is a unique $\lambda=\left(\lambda_{n}\right) \varepsilon H$ such that $\phi=\phi_{\lambda}$, that is, $\phi(\beta)=\lambda_{0} \rho+\sum_{n=1}^{\infty} \lambda_{n}\left(\rho-\beta_{n-1}\right)$ for any $B=\left(B_{n}\right) \varepsilon J$. Moreover $\|\phi\|_{J *}=\|\lambda\|_{H}$. Conversely if $\phi=\phi_{\lambda}$ then $\phi \varepsilon J J_{\text {. }}$ So that the mapping $\psi: H+J *$ defined by $\psi(\lambda)=\phi_{\lambda}$ is an isometric 1somorphism. Here, $p=11 \mathrm{~m} B$.

Proof: By the observation preceding the theorem we see that it remains to prove the first part. Let $\phi \varepsilon \mathrm{J}^{*}$; then in fact using Theorem 2.7 $\phi(B)=\phi\left(L^{-1} \circ L(B)\right)=\left(\phi \circ L^{-1}\right)(L(B))=\left(\phi \circ L^{-1}\right)\left(\left(\beta_{n}-\beta_{n-1}\right)_{n=0}^{\infty}\right), B_{-1}=0$. Now notice that $\phi \circ \mathrm{L}^{-1} \varepsilon \ell_{1}^{\star}$, so that there is a $\mu=\left(\mu_{n}\right) \varepsilon \ell_{\infty}$ such that (4.3) $\phi(\beta)=\left(\phi \circ L^{-1}\right)\left(\left(\beta_{n}-\beta_{n-1}\right)_{n=0}^{\infty}\right)=\sum_{n=0}^{\infty} \mu_{n}\left(\beta_{n}-\beta_{n-1}\right)$. By Theorem $2.8 \mu_{n}=\sum_{j=0}^{n} \lambda_{j}$ for $n \geq 0$ and some $\lambda=\left(\lambda_{j}\right) \varepsilon H$; consequently (4.3) becomes $\phi(B)=\sum_{n=0}^{\infty}\left(\sum_{j=0}^{n} \lambda_{j}\right)\left(\beta_{n}-\beta_{n-1}\right)$. Observe now that

$$
\sum_{n=0}^{N}\left(\sum_{j=0}^{n} \lambda_{j}\right)\left(\beta_{n}-\beta_{n-1}\right)=\lambda_{0} \beta_{0}+\sum_{n=1}^{N}\left(\sum_{j=0}^{n} \lambda_{j}\right)\left(\beta_{n}-\beta_{n-1}\right)=
$$




$$
\begin{aligned}
& =\lambda_{0} \beta_{N}+\sum_{n=1}^{N} \lambda_{n}\left[\beta_{N}-\beta_{n-1}\right]=\lambda_{0} \beta_{N}+\sum_{n=1}^{N} \lambda_{n}\left[\beta_{N}-\rho+\rho-\beta_{n-1}\right]= \\
& =\lambda_{0} \rho+\left[\beta_{N}-\rho\right] \sum_{n=0}^{N} \lambda_{n}+\sum_{n=1}^{N} \lambda_{n}\left[\rho-\beta_{n-1}\right] . \text { Taking the 11mit as } N \rightarrow \infty
\end{aligned}
$$

we get

$$
\sum_{n=0}^{\infty}\left(\sum_{j=0}^{\infty} \lambda_{j}\right)\left(\beta_{n}-\beta_{n-1}\right)=\lambda_{0} \rho+\sum_{n=1}^{\infty} \lambda_{n}\left[\rho-\beta_{n-1}\right]
$$

since $\left[\beta_{N}-\rho\right] \sum_{n=0}^{N} \lambda_{n} \rightarrow 0$ as $N \rightarrow \infty \quad(\rho=11 \mathrm{~m} \beta)$. Therefore we have

$\phi(\beta)=\lambda_{0} \rho+\sum_{n=1}^{\infty} \lambda_{n}\left[\rho-\beta_{n-1}\right]$.

Aga in by Theorem $2.8\left\|\phi \circ L^{-1}\right\|_{\ell \star}=\|\mu\|_{\ell}=\sup _{n>0}\left|\mu_{n}\right|=\sup \left|\sum_{j=0}^{n} \lambda_{j}\right|=\|\lambda\|_{H}$ or $\|\lambda\|_{\mathrm{H}}=\left\|\phi \circ \mathrm{L}^{-1}\right\|_{\ell_{1}^{*}} \leq\|\phi\|_{\mathrm{J} *} \cdot\left\|\mathrm{L}^{-1}\right\| \leq\|\phi\|_{\mathrm{J} *} \operatorname{since}\left\|\mathrm{L}^{-1}\right\| \leq 1$. On the other hand Theorem 4.1 gives us that $|\phi(\beta)| \leq\left\|\lambda H_{H}\right\| \beta \|_{J}$ (since $\phi=\phi_{\lambda}$ ), which implies $\|\phi\|_{\mathrm{J}} \leq\|\lambda\|_{H^{*}}$ Putting together these two inequalities we have $\|\phi\|_{\mathrm{J}}=\|\lambda\|_{\mathrm{H}}$, so that the theorem is proved.

5. CHARACTERIZATION OF OPERATORS ON S

In this section we characterize all bounded linear operators on $S$, even though an alternate proof can be given using the uniform boundedness principle. We rather use the characterization of operators on $c_{0}$. In fact we have the following result.

THEOREM 5.1. The linear mapping $A: S+c_{0}$ is bounded if and only if there is an infinite matrix $\left(a_{n k}\right)$ such that for any $s=\left(s_{n}\right) \varepsilon s$

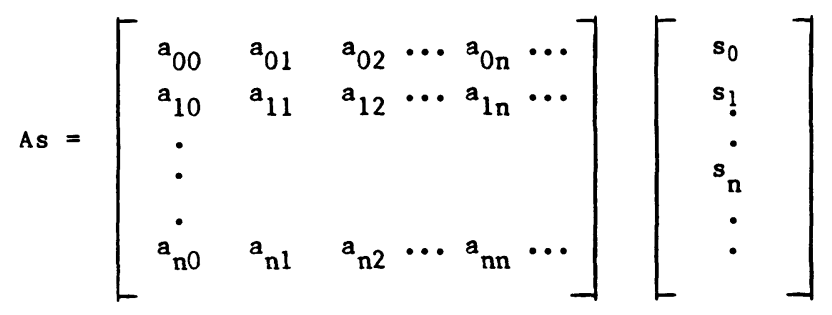

satisfying

1) $\lim _{n \rightarrow \infty} a_{n_{k}}=0$, for any fixed $k \geq 0$ 1.e. the columns of the matrix are $1 n c_{0}$,

11) $\sup _{n \geq 0} \sum_{k=0}^{\infty}\left|a_{n(k-1)}-a_{n k}\right|<\infty \quad \begin{aligned} & \text { 1.e. the rows of the matrix are } \\ & \text { uniformly bounded in } J .\end{aligned}$ Here $a_{n(-1)}=0$ for $n=0,1,2,3, \ldots$.

Proof: Note that the operator $\mathrm{P}_{\mathrm{T}^{-1}}=\mathrm{AT}_{\mathrm{A}}^{-1}$ where $\mathrm{T}^{-1}$ is as in Theorem 2.3 maps $c_{0}$ Into $c_{0}$, that is $\mathrm{P}: \mathrm{c}_{0} \rightarrow \mathrm{S}+\mathrm{c}_{0}$, so that applying Theorem 2.5 we get the desired result.

THEOREM 5.2. The 11near mapping $B: S+S$ is bounded if and only if $B$ is represented by an infinite matrix such that for some $A: S \rightarrow c_{0}$ we have 
$B=A-\tilde{A}$, where $\tilde{A}$ is defined by $\tilde{A}=\left(\dot{a}_{n k}\right)$ with $\tilde{a}_{n k}=a_{(n+1) k}$.

$\left(\tilde{A}\right.$ is a shifting of $A$ up by one row) Note that $a_{n k}=\sum_{j=n}^{\infty} b_{j k}$.

Proof: We define $A: S \stackrel{B}{T} c_{0}$, where $T$ 1s as in Theoren 2.3. That is, $A=T B$ so that $B=T^{-1} A$.

Now using the matrix representation for $\mathrm{T}^{-1}$ we get

$$
B=\left[\begin{array}{rrrrrr}
1 & -1 & 0 & 0 & 0 & \ldots \\
0 & 1 & -1 & 0 & 0 & \ldots \\
0 & 0 & 1 & -1 & 0 & \cdots \\
\cdot & \cdot & \cdot & \cdot & \cdot & \\
\cdot & \cdot & \cdot & \cdot & \cdot & \\
\cdot & \cdot & \cdot & \cdot & \cdot
\end{array}\right] A=[I-\tilde{I}] A=I A-\tilde{I} A=A-\tilde{A} \quad \text { where }
$$

I is the 1dentity matrix and $\tilde{I}$ is formed by the shifting of $I$ up by one row.

Notice Theorem 5.2 is equivalent to saying that $B: S+S$ is a bounded operator If $B$ is an infinite matrix such that each column of $B$ is summable, and if we consider the matrix $r(B)$ with $r(B)=\left(y_{n k}\right)$, where $y_{n k}=\sum_{j=n}^{\infty} b_{j k}$, then then the rows of $r(B)$ are uniformly bounded in $J$.

THEOREM 5.3. The linear mapping $C: c_{0} \rightarrow S$ is bounded if and only if $C$ is represented by an infinite matrix such that for some $M: c_{0} \rightarrow c_{0}$ we have $C=M-\tilde{M}, \tilde{M}$ is a shifting of $M$ up by one row.

Proof: Notice $c_{0} \oint_{S} f c_{0}, T$ as in Theorem 2.3. So define $M=T C$; therefore $C=T^{-1} M=M-\tilde{M}$.

Note here that the $M^{\prime} s$ are spectal $A^{\prime} s$ as are the $B^{\prime} s$. The $C^{\prime} s$ are spectal $B^{\prime} s$. The reader might wish to compare these last three theorems with Exercises 45 and 46, pg. 77, of [1].

We would 1ike to point out that the spaces $H$ and $J$ may also be found in [1] (pg. 240). Another space there, denoted by cs, is the space of all summable sequences $s=\left(s_{j}\right)$ normed with norm on $H, \max _{n \geq 0}\left|\sum_{j=0}^{n} s_{j}\right|$. It may be shown, as pointed out there, that cs* looks like J. We hasten here to note that $J$ is not the dual of cs in the sense of Definition 2.1. In particular, the inequality $\left|\sum_{j=0}^{\infty} s_{j} B_{j}\right|<\|s\|_{c s} \cdot\|B\|_{J}$ does not hold. Consider the example: Let $s=\left(s_{n}\right)$ be given by $s_{0}=1, s_{1}=s_{2}=s_{3}=s_{4}=0, s_{5}=-2, s_{6}=s_{7}=\ldots=0 ;$ also, $B=\left(\beta_{n}\right)$ : $B_{0}=0, B_{1}=-1, B_{2}=-2, B_{3}=-3, B_{4}=-4, B_{5}=B_{6}=\ldots=-4$. Notice that $\sum_{j=0}^{\infty} s_{j} \beta_{j}=8,\|B\|_{J}=4$ and $\|s\|_{c s}=\max _{n \geq 0}\left|\sum_{j=0}^{n} s_{j}\right|=1$. Therefore $\left.\left|\sum_{j=0}^{\infty} s_{j} \beta_{j}\right| \leq \max _{n \geq 0}\left|\sum_{j=0}^{n} s_{j}\right|\right] \cdot\|\beta\|_{J}$ does not hold.

To 1llustrate further the difference between cs and $S$, we note that cs and $S$ are equivalent Banach spaces: if $s$ is in $S$ then $\|s\|_{c s} \leq 2\|s\|_{S}$ and $\|s\|_{S} \leq 2\|s\|_{c s}$. So that $\phi$ is in $S^{\star}$ if and only if $\phi$ is in cs*. For $f \geq 0$ let $e_{j}$ be the sequence $\tau$ such that $\tau_{j}=1$ and 1 f $k \neq f \tau_{k}=0$. 
Now for $\phi$ in $S^{*}, \phi(s)=j_{j=0} s_{j} \phi\left(e_{j}\right)$; the sequence $\left\{\phi\left(e_{j}\right)\right\}_{j=0}^{\infty}$ is in $J$ and $\left\|\left\{\left(e_{j}\right)\right\}\right\|_{J}=\|\phi\|_{S}{ }^{*}$. But if $B$ is the sequence of the example and $\phi$ is defined on $S$ by $\phi(s)=\sum_{j=0}^{\infty} \beta_{j} s_{j}$ (so that $B_{j}=\phi\left(e_{j}\right)$ ) then $\|B\|_{J}=4$. So the natural association of $\phi$ in $c^{*}$ with the sequence $\left\{\phi\left(e_{i}\right)\right\}$ in $J$ is not an isometry. There ts an isometry from cs* onto $J$, obtained by noting that cs is isometrically isomorphic to the space $c$ of all convergent sequences normed with norm in $\ell_{\infty}$; the dual $c^{*}$ being isometrically isomorphic to $\ell_{1}$. This representation of $c^{*}$ as $\ell_{1}$, and also the representation of cs* as J, is complicated by the fact that the sequence $\left\{e_{j}\right\}$ does not have dense span in c. However, the latter representation (of $\operatorname{cs}^{\star}$ as $\mathrm{J}$ ) is made somewhat simpler by applying Thenrems 2.7 and 3.5. For $s$ in $S$ let $S_{N}(s)=\sum_{j=0}^{N} s_{j}$, the Nth partial sum of $s$, and $\sigma(s)=\sum_{j=1}^{\infty} s_{j}$. Let $\phi$ be in cs* with $\phi\left(e_{j}\right)=B_{j}$ Now, with $L$ as in Theorem 2.7, we have $\phi(s)=\sum_{j=0}^{\infty} s_{j} \phi\left(e_{j}\right)=\sum_{j=0}^{\infty} s_{j}=$ $\beta_{0} r_{0}(s)+\sum_{n=1}^{\infty} r_{n}(s)\left[\beta_{n}-\beta_{n-1}\right]=B_{0} \sigma(s)+\sum_{n=1}^{\infty}\left[\sigma(s)-s_{n-1}(s)\right]\left[\beta_{n}-\beta_{n-1}\right]=$ $\sigma(s) \nu_{0}+\sum_{n=1}^{\infty} s_{n-1}(s) \nu_{n}\left(\nu_{0}=11 m \beta, \nu_{n}=\beta_{n-1}-\beta_{n}\right) ;$ so $\nu=L(\mu)$, with $\mu_{0}=11 \mathrm{~m} B, \mu_{n}=11 \mathrm{~m} B+\beta_{0}-\beta_{n}$. These computations suggest that if one uses (as in [1]) the space cs rather than $S$ the norm to be used in $J$ should be glven by $\|\beta\|=|11 \mathrm{~m} \beta|+\sum_{n=1}^{\infty}\left|\beta_{n}-\beta_{n-1}\right| \cdot$ However, the norm used there is the same as ours (see 1, pg. 239). With this norm, $B \|$, on J, the Holder's type inequality we get is $\left|\sum_{j=0}^{\infty} \alpha_{j} \beta_{j}\right| \leq\|\alpha\|$ cs $\|\beta\|, \alpha$ in $S$ and B in J.

The reader might wish to compare the remarks of this last paragraph with some of D. J. H. Garling in pages $999-1000$ of [2] and page 964 of [3].

These Ideas were suggested by a norm on certain power series spaces introduced by the latter author In an investigation [4] of Fourier sine series and Lidstone series.

\section{REFERENCES}

[1] DUNFORD, N. and SCHWARTZ, J. T., Linear Operators, Part I, Interscience, New York, 1958.

[2] GARLING, D. J. H., On Topological Sequences Spaces, Proc. Camb. Phil. Soc. 63. (1967), 997-1019.

[3] GARLING, D. J. H., The B and y-duality of Sequences Spaces, Proc. Camb. Phil. Soc. 63 (1967), 963-981.

[4] GolightLY, George 0., Sine Series on $[0, \ell]$ for Certain Entire Functions and Lidstone Series - To appear in J. Math. Anal and Applica.

[5] ZYGMUND, A., Trigonometric Series, Cambridge University Press, London, 1959. 


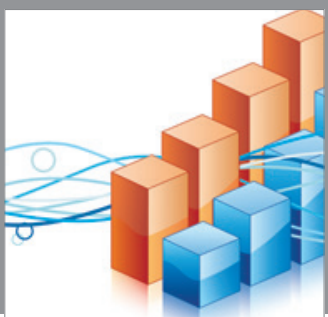

Advances in

Operations Research

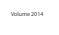

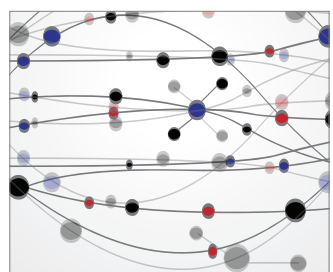

\section{The Scientific} World Journal
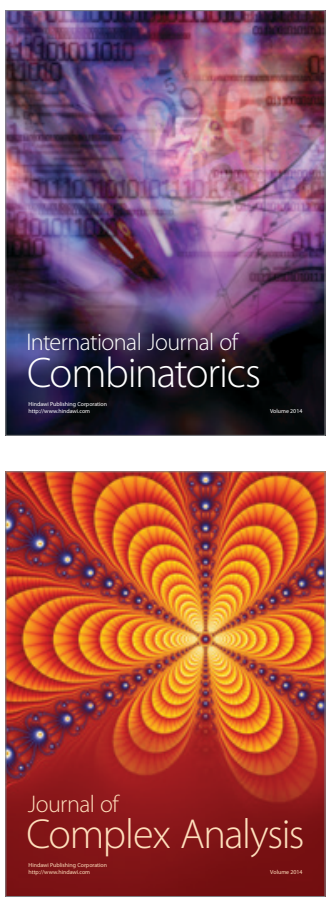

International Journal of

Mathematics and

Mathematical

Sciences
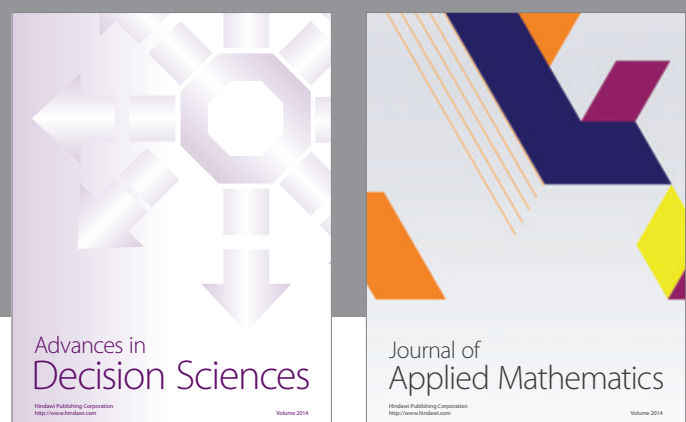

Journal of

Applied Mathematics
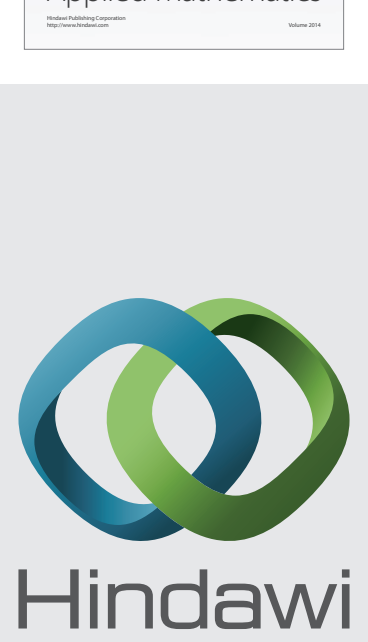

Submit your manuscripts at http://www.hindawi.com
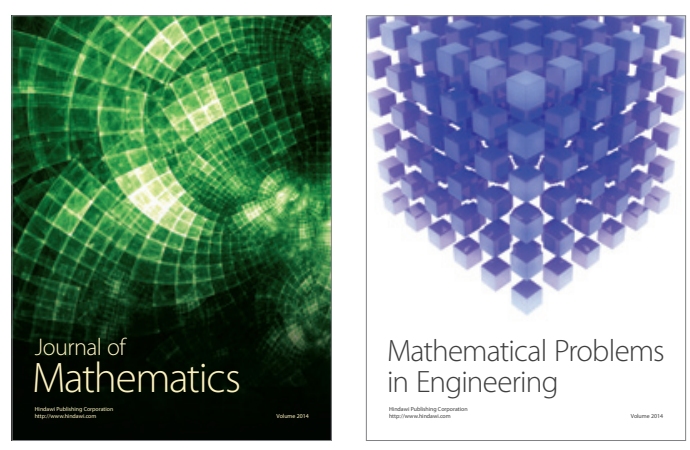

Mathematical Problems in Engineering
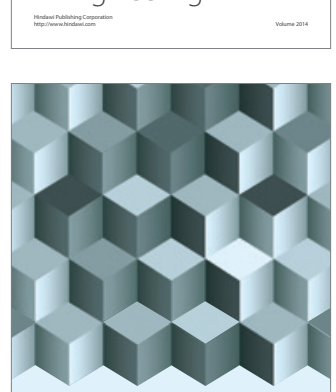

Journal of

Function Spaces
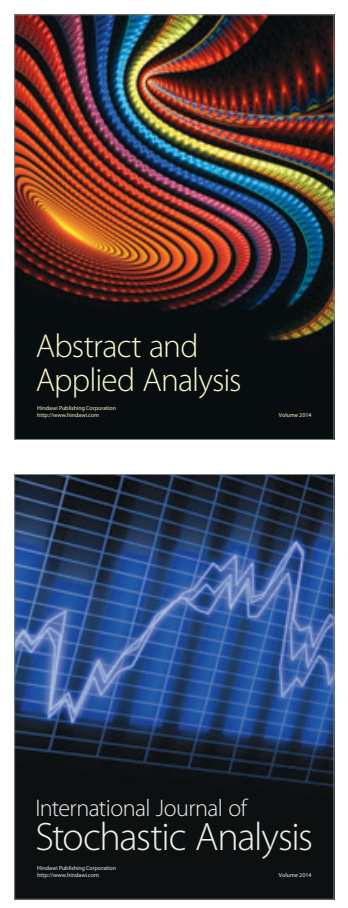

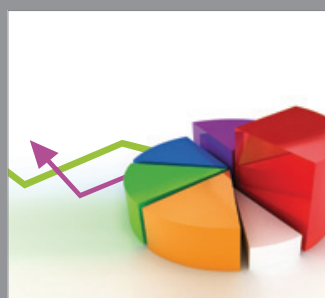

ournal of

Probability and Statistics

Promensencen
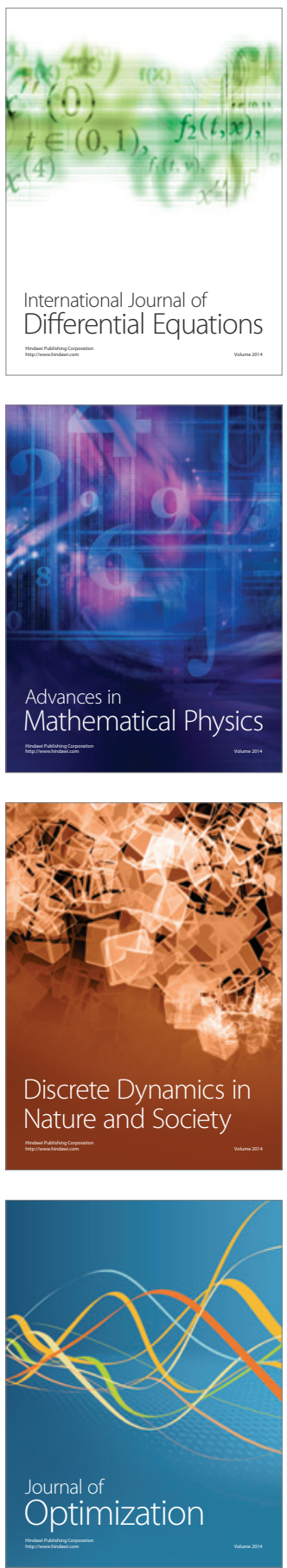